\title{
A Search for Variability in the Active T dwarf 2MASS $1237+6526$
}

\author{
Adam J. Burgasser ${ }^{1,2}$, James Liebert ${ }^{3}$, J. Davy Kirkpatrick ${ }^{4}$, and John E. Gizis ${ }^{5}$
}

\begin{abstract}
We present spectroscopic and imaging observations of the active T dwarf 2MASS 1237+6526, intended to investigate the emission mechanism of this cool brown dwarf. The $\mathrm{H} \alpha$ emission line first detected in 1999 July appears to be persistent over 1.6 years, with no significant variation from $\log _{10}\left(\mathrm{~L}_{H \alpha} / \mathrm{L}_{b o l}\right)=-4.3$, ruling out flaring as a possible source. The relatively high level of emission in this object appears to be unique amongst observed late-L and $\mathrm{T}$ dwarfs. One of our spectra shows an apparent velocity shift in the $\mathrm{H} \alpha$ line, which could be indicative of an accretion hot spot in orbit around the brown dwarf; further confirmation of this shift is required. J-band monitoring observations fail to detect any significant variability (e.g., eclipsing events) at the $\pm 0.025 \mathrm{mag}$ level over periods of up to 2.5 hours, and there appears to be no statistical evidence of variability for periods of up to 14 hours. These limits constrain the mass of a hypothetical interacting secondary to $\mathrm{M}_{2} \lesssim 20 \mathrm{M}_{J u p}$ for inclinations $i \gtrsim 60^{\circ}$. While our observations do not explicitly rule out the binary hypothesis for this object, it does suggest that other mechanisms, such as youthful accretion, may be responsible.
\end{abstract}

Subject headings: binaries: general — infrared: stars — stars: activity — stars: individual (2MASSI J1237392+652615) — stars: low mass, brown dwarfs — stars: variables: general

\section{Introduction}

A previously undetected population of $\mathrm{T}$ dwarfs, brown dwarfs exhibiting $\mathrm{CH}_{4}$ absorption bands at 1.6 and $2.2 \mu \mathrm{m}$ (Kirkpatrick et al. 1999; Burgasser et al. 2002a), have recently been identified by various deep optical and near-infrared surveys (Strauss et al. 1999; Burgasser et al. 1999, 2000b,c, 2002a; Cuby et al. 1999; Tsvetanov et al. 2000; Leggett et al. 2000; Geballe et al. 2002). One of these objects, 2MASSI J1237392+652615 (Burgasser et al. 1999, hereafter, 2MASS 1237+6526), identified in the Two Micron All Sky Survey (Skrutskie et al. 1997, hereafter 2MASS), was

\footnotetext{
${ }^{1}$ Division of Physics \& Astronomy, University of California at Los Angeles, Los Angeles, CA, 90095-1562; adam@astro.ucla.edu

${ }^{2}$ Hubble Postdoctoral Fellow

${ }^{3}$ Steward Observatory, University of Arizona, Tucson, AZ 85721; liebert@as.arizona.edu

${ }^{4}$ Infrared Processing and Analysis Center, M/S 100-22, California Institute of Technology, Pasadena, CA 91125; davy@ipac.caltech.edu

${ }^{5}$ Sharp Lab, Department of Physics \& Astronomy, University of Delaware, Newark, DE 19716; gizis@udel.edu
}

found to have $\mathrm{H} \alpha$ in emission, with a relative luminosity $\log _{10}\left(\mathrm{~L}_{H \alpha} / \mathrm{L}_{b o l}\right)=-4.3$ (Burgasser et al. 2000a). While many low-mass stars and young brown dwarfs are seen to exhibit this feature, likely generated by chromospheric magnetic activity, the frequency and relative luminosity of $\mathrm{H} \alpha$ emission drops rapidly beyond spectral types M7 V (Kirkpatrick et al. 2000; Gizis et al. 2000). Indeed, at the time of its detection, the emission of 2MASS $1237+6526$ was unique amongst objects later than type L5 V. Kirkpatrick et al. (2002) have recently reported weak $\mathrm{H} \alpha$ emission in the bright $\mathrm{T} 2 \mathrm{~V}$ SDSSp J125453.90-012247.4 (Leggett et al. 2000, hereafter SDSS 1254-0122).

In order to explain the activity of 2MASS $1237+6526$, Burgasser et al. (2000a) have hypothesized that this object may actually be an interacting brown dwarf binary system. In this scenario, the binary mass ratio and orbital separation are such that the lower-mass secondary fills its Roche lobe and steadily loses mass to the primary. For a $70 \mathrm{M}_{J u p}{ }^{6}$ primary and mass ratio

${ }^{6} \mathrm{We}$ adopt the definitions $\mathrm{M}_{J u p}=1.90 \times 10^{30} \mathrm{~g}=$ 
$q \equiv M_{2} / M_{1}>0.07$ (i.e., $M_{2}>5 \mathrm{M}_{J u p}$ ), sustained mass loss can occur for physical separations $a \lesssim 6$ $\mathrm{R}_{J u p}$ and orbital periods $p \lesssim 5.5$ hours. The close orbit required for this scenario currently rules out direct imaging of the binary pair $\left(a<00^{\prime \prime} 0002\right.$ assuming a distance of 14 pc; Burgasser et al. 1999); however, partial eclipsing could be observed in a system with an inclination $i \gtrsim 60^{\circ}$.

We have obtained followup spectroscopic and photometric observations of 2MASS $1237+6526$ in order to test the binary hypothesis, as well as examine the possibility that this object was originally observed during a prolonged flare. In $\S 2$, we present red optical (6300-10100 §) spectral data obtained over 1.6 yr using the Keck 10m Low Resolution Imaging Spectrograph (Oke et al. 1995, hereafter LRIS), and discuss the behavior of the $\mathrm{H} \alpha$ emission line over this period. J-band monitoring observations obtained over two nights using the Palomar 60" Near-Infrared Camera (Murphy et al. 1995, hereafter IRCam) are presented in $\S 3$. We discuss how the imaging observations constrain the binary scenario in $\S 4$. Finally, in $\S 5$ we discuss how these follow-up observations constrain various emission mechanisms for this unique object.

\section{Spectroscopic Observations}

2MASS $1237+6526$ has been observed on seven separate occasions in the red optical regime starting in 1999 July. Early data, obtained on 1999 July 16-18 (UT) using LRIS, are described in Burgasser et al. (2000a). Here, we present our latest LRIS observations obtained on 2000 March 5 and 2001 February 20 (UT). Data acquisition and reduction procedures for the first run are described below, and are similar to those used for the 2001 February data as described in Kirkpatrick et al. (2002).

\subsection{Data Acquisition and Reduction}

Conditions during our 2000 March 5 (UT) observations of 2 MASS $1237+6526$ were clear with 0." $8-0.99$ seeing. Because of its optical faintness (R ¿26; Matthews et al. 1996, Burgasser et al. 1999), the target was invisible on the guider camera, so

$9.55 \times 10^{-4} \mathrm{M}_{\odot}, \mathrm{R}_{\text {Jup }}=7.15 \times 10^{9} \mathrm{~cm}=0.10 \mathrm{R}_{\odot}(\mathrm{Cox}$ 2000; Tholen, Teiffel, \& Cox 2000). acquisition into a $1^{\prime \prime}$ slit was done by blind offset from a nearby optical source using positions determined from 2MASS coordinates. Spectral data were obtained using the 400 lines $\mathrm{mm}^{-1}$ grating blazed at $8500 \AA$, covering the spectral range 6300 to $10100 \AA$ at $9 \AA$ resolution. The OG570 order blocking filter was used to reduce secondorder light. Two exposures of 1800 s each were obtained; however, the data from the first exposure were fainter than expected, so the target was repositioned prior to the second exposure. Observations of the DZA4 white dwarf WD 1225-079 (Green, Schmidt, \& Liebert 1986; Kilkenny 1986) and the B1 V flux standard Hiltner 600 (Hiltner 1956; Hamuy et al. 1994) were also obtained for calibration using the same instrumental configuration.

Science data were reduced using standard $\mathrm{IRAF}^{7}$ routines. A series of one-second dark exposures were median-combined and subtracted from the science data to remove detector bias. Median-combined quartz-lamp flat-field exposures (reflected off of the interior dome) were used to normalize the response of the detector. Spectra were then extracted using the APEXTRACT routine. Curvature of the dispersion line was determined from bright standard star exposures and applied to the extraction of the 2MASS $1237+6526$ spectrum. Wavelength calibration was done using $\mathrm{Ne}-\mathrm{Ar}$ arc lamp exposures taken immediately after the target observations. We then computed a telluric correction from the white dwarf spectrum by interpolating over telluric $\mathrm{O}_{2}(6850-6900$ $\AA$ A-band, 7600-7700 $\AA$ B-band) and $\mathrm{H}_{2} \mathrm{O}$ (71507300, 8100-8350, and 9300-9650 А) bands, and we applied this correction to our flux standard and 2MASS $1237+6526$ spectra $^{8}$. Finally, flux calibration was done by correcting our Hiltner 600 observations to the spectrophotometric data given by Hamuy et al. (1994), being careful to interpolate over telluric features and the Balmer $\mathrm{H} \alpha$ line. This correction was then applied to the spectrum

\footnotetext{
${ }^{7}$ Image Reduction and Analysis Facility (IRAF) is distributed by the National Optical Astronomy Observatories, which are operated by the Association of Universities for Research in Astronomy, Inc., under cooperative agreement with the National Science Foundation.

${ }^{8}$ Despite the presence of Ca II HK absorption in this object (Kilkenny 1986; Liebert, Wehrse, \& Green 1987), the triplet lines at 8498,8542 , and $8662 \AA$ were not present and hence did not affect the telluric correction.
} 
of 2 MASS $1237+6526$.

Reduced spectral data from our 2000 March and 2001 February observations are shown in Figure 1. Full spectra with flux plotted on a logarithmic scale are shown in the right panel, while a close up of the $\mathrm{H} \alpha$ line on a linear scale is shown on the left. A combined spectrum from our 1999 July 16 (UT) observations is also plotted for comparison (Burgasser et al. 2000a). The 2000 March spectrum is roughly 2.6 times fainter than our 1999 July observations, likely the result of poor targeting onto the slit due to the blind offset and proper motion of 2MASS $1237+6526$ (first observed by 2MASS on 1999 March 13 UT). The 2001 February spectrum, on the other hand, is brighter by a factor of 1.3; atmospheric transparency on this night was excellent, and the target was visible on the facility guide camera, enabling proper slit placement. Features present in the spectra are noted, including the strong $3\left(\nu_{1}, \nu_{3}\right)$ and $2\left(\nu_{1}, \nu_{3}\right)+2 \nu_{2} \mathrm{H}_{2} \mathrm{O}$ bands starting at 9250 and $9540 \AA$ (Auman 1967), a possibly weak $4\left(\nu_{1}, \nu_{3}\right) \mathrm{CH}_{4}$ band centered at $8950 \AA$ (Dick \& Fink 1977), and lines of Cs I at 8521 and $8943 \AA$ $\left(6 s^{2} S_{1 / 2} \rightarrow 6 p^{2} P_{1 / 2,3 / 2}\right)$. The dominant feature in these spectra is the strongly pressure-broadened $\mathrm{K}$ I resonance doublet $\left(4 s^{2} S_{1 / 2} \rightarrow 4 p^{2} P_{1 / 2,3 / 2}\right)$, centered at 7665 and $7699 \AA$, but extending over most of the spectral regime shown. Liebert et al. (2000) have shown that the red wing of the K I doublet is the primary contributor to the steep slope seen from $8000-10000 \AA$ in the spectrum of SDSSp J162414.37+002915.6 (Strauss et al. 1999), and this feature is likely responsible for shaping the red optical spectrum of 2 MASS $1237+6526$ as well.

\subsection{H $\alpha$ Emission in 2MASS $1237+6526$}

$\mathrm{H} \alpha$ emission at $6563 \AA$ is clearly seen in all of the spectra of Figure 1, and its presence in our recent data suggests that emission is persistent in 2MASS $1237+6526$ over a period of at least 1.6 years. We have investigated the temporal behavior of $\mathrm{H} \alpha$ emission by measuring the line flux in all of our spectra to date. Flux data were integrated within $20 \AA$ of the emission peak after subtracting off the neighboring pseudo-continuum, the scatter of which was used to estimate uncertainties. Corrections have also been computed to compensate for slit losses, by scaling the individual spectra to match data from 1999 July 16 (UT) in the 8000$9500 \AA$ A region. The initial and corrected line fluxes are listed in Table 1.

Figure 2 plots these line fluxes versus UT time. All of the corrected values are in excellent agreement with the mean line flux from our 1999 July observations, $(7.0 \pm 0.5) \times 10^{-17} \mathrm{erg} \mathrm{cm}^{-2} \mathrm{~s}^{-1}$, with the exception of data taken on 1999 July 17 (UT). As reported by Burgasser et al. (2000a), these data were of extremely low signal-to-noise (again, likely due to poor slit placement), and it is possible that the formal errors are too low. Excepting this data point, we find that the $\mathrm{H} \alpha$ emission in 2MASS $1237+6526$ is very stable, firmly ruling out any kind of flaring event. The weighted mean value over all observations is $f_{H \alpha}=(7.44 \pm 0.08) \times 10^{-17}$ $\operatorname{erg} \mathrm{cm}^{-2} \mathrm{~s}^{-1}$, consistent with previous results.

\subsection{Comparison to Other Cool Stars and Brown Dwarfs}

To gauge how the strength of $\mathrm{H} \alpha$ emission in 2MASS $1237+6526$ compares to other active, cool dwarfs, we computed the relative $\mathrm{H} \alpha$ luminosity for each spectrum, $\log _{10}\left(\mathrm{~L}_{H \alpha} / \mathrm{L}_{b o l}\right)=$ $\log _{10}\left(f_{H \alpha} / f_{\text {bol }}\right)$, where

$$
\log _{10} f_{b o l}=-0.4 m_{b o l}-4.61
$$

in units of erg $\mathrm{cm}^{-2} \mathrm{~s}^{-1}$ (Drilling \& Landolt 2000), and $m_{b o l}=m_{J}+\mathrm{BC}_{J}$ is the apparent bolometric magnitude. Using a revised J-band magnitude for 2MASS $1237+6526$ from the 2 MASS Second Incremental Release Catalog and $\mathrm{BC}_{J}=$ $2.09 \pm 0.10$ (Leggett et al. 1999, determined from measurements of Gliese $229 \mathrm{~B}^{9}$ ), we derive $m_{b o l}=$ $18.12 \pm 0.14$ and a mean $\log _{10}\left(\mathrm{~L}_{H \alpha} / \mathrm{L}_{b o l}\right)=-4.3$, consistent with the results of Burgasser et al. (2000a).

We compare this value to the emission ratios of late-M, L, and $\mathrm{T}$ dwarfs in Figure 3. Using spectral data from Kirkpatrick et al. (1999, 2000, 2002) and Burgasser et al. (2000a, 2002b), we computed $\mathrm{H} \alpha$ line fluxes directly from the spectra as described above, with bolometric fluxes for late-M and L dwarfs computed from 2MASS J-band photometry and $\mathrm{BC}_{J}$ values from Reid et al. (2001).

\footnotetext{
${ }^{9}$ Gliese 229B and 2MASS $1237+6526$ have the same spectral type, T6.5 V (Burgasser et al. 2002a); hence, differences in the bolometric correction are likely to be negligible.
} 
For T dwarfs, we used 2MASS J-band photometry and $\mathrm{BC}_{J}=2.09$, yielding an upper limit to the true bolometric correction for the early- and mid-T dwarfs (Burgasser et al. 2002a), and hence a lower limit to the bolometric flux; i.e., overestimating the relative $\mathrm{H} \alpha$ luminosity. Detections are plotted as open symbols, while upper limits (determined from pseudocontinuum noise levels) are indicated by downward arrows. For comparison, we have included $\mathrm{M}$ and $\mathrm{L}$ dwarf samples from Hawley, Gizis, \& Reid (1996, crosses) and Gizis et al. (2000, diamonds). The trend of decreasing activity beyond M5-M7 V reported by Gizis et al. (2000) is readily apparent in this plot, and appears to continue well into the $\mathrm{T}$ dwarf regime. Indeed, only two objects have reliable detections beyond spectral type L5 V, the T2 V SDSS 12540122 , with $\log _{10}\left(\mathrm{~L}_{H \alpha} / \mathrm{L}_{b o l}\right)=-5.7$, and 2MASS $1237+6526$. The former object is over 25 times less luminous in $\mathrm{H} \alpha$ then 2MASS $1237+6526$, and upper limits for the other $\mathrm{T}$ dwarfs do not exceed $\log _{10}\left(\mathrm{~L}_{H \alpha} / \mathrm{L}_{b o l}\right)=-5.2$.

Burgasser et al. (2000a) compares 2MASS $1237+6526$ to another unusually active cool dwarf, the M9.5 Ve PC 0025+0047 (Schneider et al. 1991), which is indicated in Figure 3 by the solid square. This object, with $\log _{10}\left(\mathrm{~L}_{H \alpha} / \mathrm{L}_{b o l}\right)=-3.4$, is also more than ten times brighter in $\mathrm{H} \alpha$ than other objects in its class, although it is not extremely active in comparison to earlier emission M dwarfs (Hawley, Gizis, \& Reid 1996). PC $0025+0047$ also shows long-term stability in its emission (Schneider et al. 1991; Mould et al. 1994; Martín, Basri, \& Zapatero Osorio 1999). These common characteristics provide further evidence that emission processes in these two objects may be related.

\subsection{A Possible Velocity Shift in the 2000 March Spectrum?}

Examination of the spectra of Figure 1 also shows an apparent blueshift in the $\mathrm{H} \alpha$ line in our 2000 March data as compared to the other epochs. We measured a line center deviation of $3.2 \pm 0.5 \AA$ in this spectrum with respect to the 2001 February data, where the uncertainty is derived from the scatter of telluric $\mathrm{OH}$ emission line wavelengths between these two data sets. This deviation corresponds to a velocity of $150 \pm 25 \mathrm{~km} \mathrm{~s}^{-1}$, which cannot be due to differences in the Earth's rota- tional and orbital motion between the two epochs, amounting to only $2.4 \mathrm{~km} \mathrm{~s}^{-1}$.

As with our initial detection of $\mathrm{H} \alpha$, we carefully scrutinized the reality of this subtle (1-2 pixel) line shift. Figure 4 shows the $\mathrm{H} \alpha$ region in the raw image data from 2000 March and 2001 February. Telluric OH emission lines in the $6563 \AA$ region appear as vertical bands, while the bright spots are $\mathrm{H} \alpha$ emission in 2MASS $1237+6526$; note the lack of continuum in this region. There is a slight offset in the emission between these two epochs with respect to the telluric lines, confirming the spectral results in Figure 1. However, this shift could be due to poor centering of the object on the slit. Both individual exposures taken on 2000 March 5 (UT) show a similar offset, despite repositioning of the object. The second exposure was brighter and probably better positioned, but was still 2.6 times fainter than our 1999 July results; hence, it too may have been misaligned. On the other hand, seeing on the nights of 2000 March 5 and 2001 February 20 (UT) was roughly equivalent to the width of the slit employed, so significant shifting of the spectrum due to poor slit placement is less likely. We were unable to perform an independent check using the weak 8521 and $8943 \AA$ Cs I lines due to the low signal-to-noise of both spectra. We therefore conclude that there is some evidence for a velocity shift in the $\mathrm{H} \alpha$ line which must be confirmed with higher resolution data.

Assuming its reality, the $\mathrm{H} \alpha$ line shift (which appears to be asymmetric toward the blue) could arise from a single hot spot on the surface of a rapidly rotating brown dwarf. The velocity of the line is comparable to but less than the break-up speed for a substellar object, roughly $330(520) \mathrm{km}$ $\mathrm{s}^{-1}$ for a $30(75) \mathrm{M}_{J u p}$ brown dwarf. In this case, however, the integration time of each individual observation made (typically 20-30 minutes) would be a significant fraction of the rotation period (no less than about 50 minutes), and one would expect the line to be broadened rather than shifted. A more reasonable source would be a hot spot in orbit around the brown dwarf, possibly arising from an optically thin accretion disk. Discrete regions in an edge-on Keplerian disk would have line-ofsight radial velocities as high as $150 \mathrm{~km} \mathrm{~s}^{-1}$ at separations of roughly 5 (12) $\mathrm{R}_{\text {Jup }}$ for a 30 (75) $\mathrm{M}_{\text {Jup }}$ central source. The hot spot would have to be small, due to the lack of blue continuum seen in 
at shorter wavelengths (Burgasser et al. 2000a). It is worth noting that the magnitude and direction of the velocity shift is similar to that seen in some solar flaring events during an impulsive evaporation phase (Antonucci et al. 1985); however, we do not see the typically brighter redshifted condensation component (Fisher, Canfield, \& McClymont 1985).

\section{Imaging Observations}

In addition to our spectroscopic data, we searched for photometric variability in 2MASS $1237+6526$, for the purpose of detecting an eclipse by a closely-separated companion.

\subsection{Data Acquisition and Reduction}

J-band monitoring observations were obtained over two nights, 2000 May 19-20 (UT), using the Palomar 60" IRCam. Conditions on both nights were generally hazy but clear, with average seeing of 1 .'2 to 1 ". 8 ; some scattered cirrus was present during the observations of May 20. Data were acquired in sets of five 45s exposures dithered by $10^{\prime \prime}$ on May 19 and $15^{\prime \prime}$ on May 20 in a square pattern. A total of 155 and 165 images were obtained, spanning periods of 2.50 and 2.57 hours on May 19 and 20 , respectively (Table 2 ).

Science data were initially pairwise differenced to eliminate bias and sky background, and then divided by a normalized flat-field image, constructed by differencing median-combined sets of lamp-on and lamp-off dome observations made on May 19. Bad pixels were identified in the flat-field image and corrected in the science images by interpolation. The four separate readouts of the IRCam NICMOS-3 HgCdTe array occasionally introduced additive quadrant biases that we removed by subtracting off the median from each quadrant. Finally, individual images in each set were shifted and coadded to produce a final reduced image (Figure 5).

\subsection{Differential Photometry and Analysis of the Time Series}

For each image, we computed aperture photometry $\left(f_{i}\right)$ for 2MASS $1237+6526$ and other sources in the field. Flux within a 2.5 (4.04 pixel) radius aperture was integrated after subtracting the mean background measured in a 6 to $12^{\prime \prime}$ annulus around each object. Uncertainty estimates of the photometry, $\delta f_{i}$, included contributions by shot noise in the source and variations in the background (essentially flat field uncertainties). Instrumental magnitudes, $m_{i}=-2.5 \log _{10} f_{i}$, were then computed with corresponding uncertainties, $\sigma_{i}=1.086\left(\frac{\delta f_{i}}{f_{i}}\right)$. The scatter in instrumental magnitudes for 2MASS $1237+6526$ was roughly 0.03 mag on May 19 and 0.01 mag on May 20, with formal photometric uncertainties of $\pm 0.025 \mathrm{mag}$.

To compute a relative magnitude light curve, we first examined sources in the field for variability, using the procedure outlined in Bailer-Jones \& Mundt (2001). We first determined relative magnitudes and uncertainties for each source:

$$
\begin{gathered}
\hat{m}_{i}^{(j)}=m_{i}^{(k)}-m_{i}^{r e f} \\
\hat{\sigma}_{i}^{(j)^{2}}=\sigma_{i}^{(j)^{2}}+\sigma_{i}^{r e f^{2}},
\end{gathered}
$$

where the reference magnitudes and uncertainties were computed as

$$
\begin{gathered}
m_{i}^{r e f}=-2.5 \log _{10}\left(\sum_{k \neq j}^{K} f_{i}^{(k)}\right) \\
\sigma_{i}^{r e f^{2}}=\sum_{k \neq j}^{K} \sigma_{i}^{(k)^{2}},
\end{gathered}
$$

summing over the $K$ other sources in the field. After subtracting off the mean relative magnitude, we computed the reduced $\chi^{2}$ for each source to determine if photometric variations were significant:

$$
\chi_{r}^{2}=\frac{1}{n-1} \sum_{i}^{n}\left(\frac{\hat{m}_{i}-\left\langle\hat{m}_{i}\right\rangle}{\hat{\sigma}_{i}}\right)^{2} ;
$$

here, $n$ is the total number of images. We constrained calibrator sources to have $\chi_{r}^{2} \lesssim 0.5$, corresponding to a probability of variability $P$ $\leq 1 \%$ for $n=30$. Two calibrators present in images from both nights passed this constraint, 2MASSI J1237367+652600 (Calibrator 1) and 2MASSI J1237372+652536 (Calibrator 2). These sources have 2MASS J magnitudes of $15.07 \pm 0.05$ and $15.34 \pm 0.06$, respectively, and are indicated on Figure 5 .

Relative magnitudes were then computed for 2MASS $1237+6526$ using these two calibrator stars. The resulting time series is shown for both 
nights in Figure 6. The mean relative magnitude has been subtracted off in both datasets. Time series for the differenced calibrator magnitudes $\left(m_{i}^{(1)}-m_{i}^{(2)}\right)$ are also shown (offset) for comparison. Scatter in the relative magnitudes of 2MASS $1237+6526$ was 0.020 and $0.014 \mathrm{mag}$ on May 19 and 20, respectively, less than the formal photometric uncertainties. Corresponding $\chi_{r}^{2}$ values are 0.505 and 0.314 , which are greater than those of the calibrator stars (0.271 and 0.237 , respectively), but nevertheless reject variability (at levels above our detection threshold) at the $>99 \%$ confidence level.

To examine to possibility of a modulated signal (e.g., multiple eclipsing events or geometric modulation), we constructed periodograms for each night's data using the formalism of Lomb (1976) and Scargle (1982) as summarized in Press et al. (1992). Given the duration of the observations, we are sensitive to periods ranging from roughly 0.1-1.3 hr on each night. The computed periodograms (oversampled by a factor of 10) for both 2MASS $1237+6526$ (black lines) and calibrator stars (grey lines) are shown in Figure 7 . May 19 and 20 data are identified by solid and dashed lines, respectively. None of the peaks in the 2MASS $1237+6526$ periodograms are significant, as they do not exceed the $50 \%$ false alarm probability level and are roughly equal in magnitude to insignificant peaks seen in the calibrator periodograms.

Variability over two nights also appears to be absent. There is a 0.024 mag offset in the mean relative magnitudes between the two nights; this is greater than the difference between the mean calibrator values (0.003 mag), but comparable to the photometric uncertainties. We compute $\chi_{r}^{2}=$ 0.593 over both nights, again rejecting variability at the $>99 \%$ confidence level. Because of the large temporal gap in the time series data, periodicity analysis requires more sophisticated algorithms (e.g., the CLEAN algorithm; Roberts, Léhar, \& Dreher 1987); however, given the lack of statistical evidence for variability, further analysis appears to be unwarranted.

Given the null results from these statistical tests, we conclude that there is no short-term variability in 2 MASS $1237+6526$ at the $\pm 0.025 \mathrm{mag}$ level over periods of $0.1-2.5$ hours and no evidence of periodicity for periods of up to 1.3 hours. There also appears to be no compelling indication of variability at this level for periods of up to $\sim 14$ hours.

\section{The Binary Hypothesis Revisited}

In order to determine what constraints our null variability results impose on the interacting binary hypothesis, we have updated the basic theory described in Burgasser et al. (2000a) by comparison with theoretical models from Burrows et al. (1997). Our observables are (1) a period limit from the duration of monitoring observations, (2) a magnitude limit at which we are sensitive to variability, and (3) the properties of the primary. We therefore seek to derive a relation $\Delta \mathrm{J}=f\left(p, i ; M_{1}\right)$, where $p$ and $i$ are the period and inclination of the system, and $M_{1}$ the mass of the primary. For this discussion, we normalize masses to $\mathrm{M}_{J u p}$ and distances to $\mathrm{R}_{J u p}$, and assume a circular orbit and negligible angular momentum loss (e.g., to gravitational radiation or disk interaction). The period (in hours) is determined from the standard Keplerian relation:

$$
p^{2}=4 \pi^{2} \frac{a^{3}}{G\left(M_{1}+M_{2}\right)}=8.79 \frac{a^{3}}{M_{1}(1+q)}
$$

in Jupiter-based units. In the case of an interacting binary, the secondary fills its Roche lobe, and hence its radius $R_{2}$ is determined by

$$
R_{2}=R_{L}=a \frac{0.49 q^{\frac{2}{3}}}{0.6 q^{\frac{2}{3}}+\ln \left(1+q^{\frac{1}{3}}\right)} .
$$

(Eggleton 1983). Brown dwarf radii, however, are fairly constant from 5-70 $\mathrm{M}_{\text {Jup }}$ (Burrows \& Liebert 1993), and are well-described by the relation (Zapolsky \& Salpeter 1969; Stevenson 1991):

$$
R=\alpha_{1} \frac{\pi M^{-\frac{1}{3}}}{\left(1+1.8 M^{-\frac{1}{2}}\right)^{\frac{4}{3}}},
$$

where $\alpha_{1}$ is an age-dependent scale factor (essentially a correction to partial degeneracy) chosen to match the interior models of Burrows et al. (1997). Because $R_{2}$ depends only on the primary mass and mass ratio $q$, both $a$ and $p$ are functions of $\mathrm{M}_{1}$ and $q$ exclusively. Therefore, these two parameters define the dynamics of the system.

To determine the observable change in J-band magnitude caused by a nearly edge-on eclipse, we assumed a simple geometry of overlapping spheres 
along the line of sight, as diagrammed in Figure 8. In general, because $\operatorname{dlnR} / \operatorname{dnn} M \approx-\frac{1}{3}<0$ for brown dwarfs, the lower-mass secondary has a larger radius $\left(R_{2}>R_{1}\right)$ for $M_{2}>5 \mathrm{M}_{\text {Jup }}$. The relative area of the primary, $A$, obscured by the secondary during maximal eclipse is:

$$
\pi A=\theta_{1}-0.5 \sin 2 \theta_{1}+\left(\frac{R_{2}}{R_{1}}\right)^{2}\left(\theta_{2}-0.5 \sin \theta_{2}\right)
$$

where

$$
\begin{gathered}
R_{1} \sin \theta_{1}=R_{2} \sin \theta_{2} \\
R_{1} \cos \theta_{1}+R_{2} \cos \theta_{2}=a \cos i,
\end{gathered}
$$

with $\theta_{1}$ and $\theta_{2}$ defined in Figure 8 . These equations allow us to uniquely determine $A$ in terms of $M_{1}, q$, and $i$. Note that no eclipse occurs when $\cos i>\left(R_{1}+R_{2}\right) / a$, while a complete eclipse occurs for $\cos i<\left(R_{2}-R_{1}\right) / a$.

The observed magnitude difference at maximal eclipse is then

$$
\begin{aligned}
\Delta J & =-2.5 \log _{10}\left(\frac{F_{1}(1-A)+F_{2}}{F_{1}+F_{2}}\right) \\
& =2.5 \log _{10}\left(\frac{A}{1+\beta}\right),
\end{aligned}
$$

where $\beta=F_{2} / F_{1}$ is the flux ratio of the secondary to primary at J-band. We can determine the change in J-band magnitude as a function of $\mathrm{T}_{\text {eff }}$ empirically from the two known companion T dwarfs, Gliese 229B (Nakajima et al. 1995) and Gliese 570D (Burgasser et al. 2000b). The former object is a T6.5 V with $\mathrm{M}_{J}=15.52 \pm 0.06$ (Leggett et al. 1999) and $\mathrm{T}_{\text {eff }} \sim 950 \mathrm{~K}$ (Allard et al. 1996; Marley et al. 1996; Tsuji et al. 1996); the latter object is a T8 V with $\mathrm{M}_{J}=16.47 \pm 0.07$ (Burgasser et al. 2000b) and $\mathrm{T}_{\text {eff }} \sim 800 \mathrm{~K}$ (Geballe et al. 2001). These values yield the very approximate relation $\mathrm{d} \mathrm{J} / \mathrm{dT}=-0.0063 \mathrm{mag} / \mathrm{K}$, which may only be accurate over a limited $\mathrm{T}_{\text {eff }}$ range ${ }^{10}$. Interior models from Burrows et al. (1997) show a power law relation between $\mathrm{T}_{\text {eff }}$ and mass which is roughly dependent on age $(\tau)$. A fit to the models yields the relations:

$$
\frac{d T}{d M}= \begin{cases}88.7 M^{-0.43} & \tau=0.5 \text { Gyr } \\ 73.4 M^{-0.44} & \tau=1.0 \text { Gyr } \quad \text { K/M } / M_{\text {Jup }} . \\ 45.4 M^{-0.44} & \tau=5.0 \text { Gyr }\end{cases}
$$

${ }^{10}$ This rough relation also ignores the effects of gravity and metallicity, which could be substantial.
Combining Equation 14 with the expression for $\mathrm{dJ} / \mathrm{dT}$ yields the secondary flux ratio:

$$
\begin{aligned}
-2.5 \log _{10} \beta & =\frac{d J}{d T} \frac{d T}{d M} \Delta M \\
& =\frac{d J}{d T} \frac{d T}{d M} M_{1}(q-1) \\
& =\alpha_{2} \times(1-q)
\end{aligned}
$$

where $\alpha_{2}$ is a constant dependent on age and primary mass. We have assumed that 2MASS $1237+6526$, a T6.5 V similar to Gliese 229B, has a $\mathrm{T}_{\text {eff }} \sim 950 \mathrm{~K}$, and masses 23,30 , and $55 \mathrm{M}_{\text {Jup }}$ for $\tau=0.5,1.0$, and $5.0 \mathrm{Gyr}$, respectively (Burrows et al. 1997). The various parameters are summarized in Table 3.

Using the above equations, we have calculated the expected magnitude drop $\left(\Delta \mathrm{J}_{e c l}\right)$ at minimum light for an eclipsing, interacting brown dwarf binary with $\mathrm{T}_{\text {primary }}=950 \mathrm{~K}, 0.5 \leq \tau \leq 5.0 \mathrm{Gyr}, 0$ $\leq q \leq 1$, and $40^{\circ} \leq i \leq 90^{\circ}$. Results are shown in Figure 9 for $\tau=0.5$ (solid line), 1.0 (dot-dashed line), and 5.0 (dashed line) Gyr, and (from top to bottom) $i=90^{\circ}, 80^{\circ}, 70^{\circ}$, and $60^{\circ}$. Only values of $\Delta \mathrm{J}_{\text {ecl }}$ for which $q<0.63$ and $\mathrm{M}_{2}>5 \mathrm{M}_{\text {Jup }}$ are displayed, corresponding to the limits for sustained mass loss (Burgasser et al. 2000a). Significant drops in the observed flux are predicted by this model, particularly for $i \geq 80^{\circ}$, where fluxes at maximum eclipse over 0.7 mag fainter than the nominal brightness are possible over the full range of periods shown. These flux deviations are due primarily to the substantial brightness ratio between the secondary and primary, which is particularly important at high inclination angles where obscuration is most complete. At lower inclination angles, the limiting factor is the orbital separation, which is larger for lower mass secondaries. No eclipse variations are predicted to occur for $i \lesssim 50^{\circ}$ over the range of ages examined. Note that significant systematic errors may be present for the lowest mass ratios (i.e., low secondary $\mathrm{T}_{\text {eff }}$ ) due to the rough estimate of $\beta$ in Equation 15; however, our observations generally sample only the highest mass ratios due to the period cutoff.

The predicted $\Delta \mathrm{J}_{e c l}$ from these models are generally much larger than our observational limits (indicated by the hatched region in Figure 8). The constraints imposed by the non-detection of variability are summarized in Table 3 for $i>60^{\circ}$; in this case, $q>0.70,0.53$, and 0.37 , corresponding to secondary masses $\mathrm{M}_{2}>16,16$, and $20 \mathrm{M}_{J u p}$ for $\tau=0.5,1.0$, and $5.0 \mathrm{Gyr}$, respectively. Note that 
for the 0.5 Gyr case, our mass ratio limit exceeds the minimum $q$ required to sustain mass loss. The lowest inclination angle eclipses that could have been detected by our observations for $\tau=1.0$ and 5.0 Gyr are $i_{\min }=54^{\circ}$ and $56^{\circ}$, with corresponding minimum secondary masses of 20 and $34 \mathrm{M}_{\text {Jup }}$, respectively.

\section{Discussion}

Based on the results of the monitoring observations, we can confidently rule out an interacting binary system for 2MASS $1237+6526$ for $i>60^{\circ}$ and $\mathrm{M}_{2}>20 \mathrm{M}_{\text {Jup }}$. We cannot rule out a less inclined system, although such a geometry would fail to explain the apparent line shift seen in our 2000 March spectral data. A compromise solution would be a moderately inclined system with a massive primary and large mass ratio. Conclusive synthesis of the spectroscopic and photometric data, however, requires verification of $\mathrm{H} \alpha$ line shift.

Other emission mechanisms should also be considered. 2MASS $1237+6526$ could be a young, and hence very low mass, brown dwarf that is still accreting material from a circum(sub)stellar disk. If this object were in a weak-lined T-Tauri (WLTT) phase, its $\mathrm{H} \alpha$ line could arise from a small, optically thin accretion boundary layer, consistent with our spectral and photometric observations. Assuming a typical WLTT age (30-100 Myr; Hartmann, Kenyon, \& Hartigan 1993) and $\mathrm{T}_{\text {eff }} \sim 950 \mathrm{~K}$, the evolutionary models of Burrows et al. (1997) predict a mass of 3-12 $\mathrm{M}_{J u p}$, below the Deuterium-burning limit. Note that 2MASS $1237+6526$ is not associated with any known star-forming region, although it could have been ejected from its nascent cluster (Reipurth \& Clarke 2001). The absence of the $1.25 \mu \mathrm{m} \mathrm{K} \mathrm{I}$ doublet in this object (I. McLean, priv. comm.) marginally supports the possibility of it being a low-gravity source, although we cannot rule out a purely temperature effect (Burgasser et al. 2002a).

Our follow-up observations of 2MASS $1237+6526$ have enabled us to rule out flaring as a viable means of emission, and have placed significant constraints on the geometry and membership of an interacting binary system. Parallax (to determine absolute brightness) and space motion measurements are clearly required to further characterize this object. Furthermore, the apparent velocity shift described in $\S 2.4$, which may prove to be a vital clue to the origin of emission in 2 MASS $1237+6526$, requires confirmation and more detailed, higher-resolution investigation. The nature of this enigmatic brown dwarf may hopefully be revealed with further observations.

A. J. B. would like to thank Telescope Operators Terry Stickel (Keck) and Barrett "Skip" Staples (Palomar) for their support during the observations presented here. A. J. B. also acknowledges useful discussions with C. A. L. BailerJones, D. Koerner, M. Marley, I. McLean, and M. R. Zapatero Osorio during the preparation of the manuscript, and valuable comments from our anonymous referee. A. J. B., J. D. K., and J. E. G. acknowledge the support of the Jet Propulsion Laboratory, California Institute of Technology, which is operated under contract with the National Aeronautics and Space Administration. A. J. B. also acknowledges support provided by NASA through a Hubble fellowship grant from the Space Telescope Science Institute, which is operated by the Association of Universities for Research in Astronomy, Incorporated, under NASA contract NAS5-26555. J. L. acknowledges support from NASA JPL grant 961040NSF. Portions of the data presented herein were obtained at the W. M. Keck Observatory which is operated as a scientific partnership among the California Institute of Technology, the University of California, and the National Aeronautics and Space Administration. The Observatory was made possible by the generous financial support of the W. M. Keck Foundation. This publication makes use of data from the Two Micron All Sky Survey, which is a joint project of the University of Massachusetts and the Infrared Processing and Analysis Center, funded by the National Aeronautics and Space Administration and the National Science Foundation.

\section{REFERENCES}

Allard, F., Hauschildt, P. H., Baraffe, I., \& Chabrier, G. 1996, ApJ, 465, L123

Antonucci, E., Dennis, B. R., Gabriel, A. H., \& Simnett, G. M. 1985, Sol. Phys., 96, 129

Auman, J., Jr. 1967, ApJS, 14, 171 
Bailer-Jones, C. A. L., \& Mundt, R. 2001, A\&A, 367,218

Burgasser, A. J., Kirkpatrick, J. D., Reid, I. N., Liebert, J., Gizis, J. E., \& Brown, M. E. 2000, AJ, 120, 473

Burgasser, A. J., et al. 2002, ApJ, in preparation

Burgasser, A. J., et al. 2002, ApJ, 564, 421

Burgasser, A. J., et al. 2000, ApJ, 531, L57

Burgasser, A. J., et al. 2000, AJ, 120, 1100

Burgasser, A. J., et al. 1999, ApJ, 522, L65

Burrows, A., \& Liebert, J. 1993, Rev. Mod. Phys. 65,301

Burrows, A., et al. 1997, ApJ, 491, 856

Cox, A. N. 2000, in Allen's Astrophysical Quantities, Fourth Edition, ed. A. N. Cox (New York: Springer-Verlag), p. 7

Cuby, J. G., Saracco, P., Moorwood, A. F. M., D’Odorico, S., Lidman, C., Comerón, F., \& Spyromilio, J. 1999, A\&A, 349, L41

Dick, K. A., \& Fink, U. 1977, JQSRT, 18, 433

Drilling, J. S., \& Landolt, A. U. 2000, in Allen's Astrophysical Quantities, Fourth Edition, ed. A. N. Cox (New York: Springer-Verlag), p. 381

Eggleton, P. P. 1983, ApJ, 268, 368

Fisher, G. H., Canfield, R. C., \& McClymont, A. N. 1985, ApJ, 289, 434

Gizis, J. E., Monet, D. G., Reid, I. N., Kirkpatrick, J. D., Liebert, J., \& Williams, R. 2000, AJ, 120, 1085

Geballe, T. R., Saumon, D., Leggett, S. K., Knapp, G. R., Marley, M. S., \& Lodders, K. 2001, ApJ, 556, 373

Geballe, T. R., et al. 2001, ApJ, 564, 466

Green, R. F., Schmidt, M., \& Liebert, J. 1986, ApJS, 61, 305

Hamuy, M., Suntzeff, N. B., Heathcote, S. R., Walker, A. R., Gigoux, P., \& Phillips, M. M. 1994, PASP, 106, 566
Hartmann, L., Kenyon, S., \& Hartigan, P. 1993, in Planets \& Protostars III, ed. E. H. Levy \& J. I. Lunine (Tucson: Univ. Arizona Press), 497

Hawley, S. L., Gizis, J. E., \& Reid, I. N. 1996, AJ, 112,2799

Hiltner, W. A. 1956, ApJS, 2, 389

Kilkenny, D. 1986, The Observatory, 106, 201

Kirkpatrick, J. D., Reid, I. N., Liebert, J., Gizis, J. E., Burgasser, A. J., Monet, D. G., Dahn, C. C., Nelson, B., \& Williams, R. J. 2000, AJ, 120,447

Kirkpatrick, J. D., et al. 2002, ApJ, in preparation

Kirkpatrick, J. D., et al. 1999, ApJ, 519, 802

Leggett, S. K., Toomey, D. W., Geballe, T. R., \& Brown, R. H. 1999, ApJ, 517, L139

Leggett, S. K., et al. 2000, ApJ, 536, L35

Liebert, J., Reid, I. N., Burrows, A., Burgasser, A. J., Kirkpatrick, J. D., \& Gizis, J. E. 2000, ApJ, 533, L155

Liebert, J., Wehrse, R., \& Green, R. F. 1987, A\&A, 175, 173

Lomb, N. R. 1976, Ap\&SS, 39, 447

Marley, M. S., Saumon, D., Guillot, T., Freedman, R. S., Hubbard, W. B., Burrows, A., \& Lunine, J. I. 1996, Science, 272, 1919

Martín, E. L., Basri, G., \& Zapatero Osorio, M. R. 1999, AJ, 118, 1005

Matthews, K., Nakajima, T., Kulkarni, S. R., \& Oppenheimer, B. R. 1996, AJ, 112, 1678

Mould, J., Cohen, J., Oke, B., \& Reid, N. 1994, AJ, 107, 2222

Murphy, D. C., Persson, S. E., Pahre, M. A., Sivaramakrishnan, A., \& Djorgovski, S. G. 1995, PASP, 107, 1234

Nakajima, T., Oppenheimer, B. R., Kulkarni, S. R., Golimowski, D. A., Matthews, K., \& Durrance, S. T. 1995, Nature, 378, 463

Oke, J. B., et al. 1995, PASP, 107, 375 
Press, W. H., Teukolsky, S. A., Vetterling, W. T., Flannery, B. P. 1992, Numerical Recipes, Second Edition (New York: Cambridge University Press)

Reid, I. N., Burgasser, A. J., Cruz, K., Kirkpatrick, J. D., \& Gizis, J. E. 2001, AJ, 121, 1710

Reipurth, B., \& Clarke, C. 2001, AJ, 122, 432

Roberts, D. H., Léhar, J., \& Dreher, J. W. 1987, AJ, 93, 968

Scargle, J. D. 1982, ApJ, 263, 835

Schneider, D. P., Greenstein, J. L., Schmidt, M., \& Gunn, J. E. 1991, AJ, 102, 1180

Skrutskie, M. F., et al. 1997, in The Impact of Large-Scale Near-IR Sky Surveys, ed. F. Garzon (Dordrecht: Kluwer), p. 25

Stevenson, D. J. 1991, ARA\&A, 29, 163

Strauss, M. A., et al. 1999, ApJ, 522, L61

Tholen, D. J., Teiffel, V. G., \& Cox, A. N. 2000, in Allen's Astrophysical Quantities, Fourth Edition, ed. A. N. Cox (New York: SpringerVerlag), p. 293

Tsuji, T., Ohnaka, K., Aoki, W., \& Nakajima, T. 1996, A\&A, 308, L29

Tsvetanov, Z. I., et al. 2000, ApJ, 531, L61

Zapolsky, H. S., \& Salpeter, E. E. 1969, ApJ, 158, 809

This 2-column preprint was prepared with the AAS LATEX macros v5.0. 
Fig. 1.- Optical spectra of 2MASS $1237+6526$ obtained using LRIS. Observations from 1999 July 16, 2000 March 5, and 2001 February 20 (UT) are shown, and are scaled by the correction factors described in $\S 2.2$. The right panel shows full red optical spectra from $6300-10100 \AA$, with flux plotted on a logarithmic scale to enhance features; spectra are offset by a constant factor. Observed bands of $\mathrm{H}_{2} \mathrm{O}$ and $\mathrm{CH}_{4}$, and lines of $\mathrm{Cs} \mathrm{I}$ and $\mathrm{H} \alpha$ are labelled, along with the pressure-broadened $\mathrm{K}$ I doublet. The left panel shows a closeup of the $\mathrm{H} \alpha$ line on a linear scale, with data offset by an additive constant (dotted lines).

Fig. 2.- Emitted $\mathrm{H} \alpha$ flux from 2MASS $1237+6526$ versus UT time. Circles plot corrected line flux values, scaled by matching flux data in the 8000-9500 $\AA$ region to observations made on 1999 July 16 (UT). The mean emission strength $f_{H \alpha}=(7.44 \pm 0.08) \times 10^{-17} \mathrm{erg} \mathrm{cm}^{-2} \mathrm{~s}^{-1}$ is indicated by the dashed line.

Fig. 3.- Relative $\mathrm{H} \alpha$ luminosity versus spectral type for cool dwarfs. The mean relative luminosity for 2MASS $1237+6526$ is indicated by the solid circle, while values calculated from late-M, L, and $\mathrm{T}$ dwarf spectra are shown as open symbols (detected) or arrows (nondetection upper limits). Also shown are data for M and L dwarfs from Hawley, Gizis, \& Reid (1996, crosses) and Gizis et al. (2000, diamonds), and for PC $0025+0047$ (Schneider et al. 1991, solid square). T spectral types are from Burgasser et al. (2002a).

Fig. 4.- Raw LRIS data of 2MASS $1237+6526$ from 2000 March 5 (top) and 2001 February 20 (UT, bottom) in the $6563 \AA$ region. Vertical bands are telluric $\mathrm{OH}$ emission lines, while the bright spots arise from $\mathrm{H} \alpha$ emission in 2MASS $1237+6526$.

Fig. 5.- Combination of five, $45 \mathrm{sec} \mathrm{J}$-band images of the 2MASS $1237+6526$ field obtained on 2000 May 19 (UT). 2MASS $1237+6526$ is indicated, as are the two comparison stars Cal 1 and Cal 2. The field shown is $2^{\prime} \times 2^{\prime}$, oriented with North up and East toward the left.

Fig. 6.- Relative magnitude time series for 2MASS $1237+6526$ (diamonds) and comparison stars (triangles). Data for 2000 May 19 and 20 (UT) are shown separately. The mean relative magnitudes for each night have been subtracted, with datasets offset for clarity.

Fig. 7.- Periodograms of monitoring time series data. Black and grey lines trace the periodograms of 2MASS $1237+6526$ and comparison stars, respectively, while solid and dashed lines identify data from 2000 May 19 and 20 (UT). False alarm probability limits of $50 \%$ and $10 \%$ are indicated.

Fig. 8.- Geometry of the interacting binary model. (a) Orientation along the line-of-sight; $a$ is the binary separation, $i$ the inclination, $M_{1}$ and $M_{2}$ the masses of the primary and secondary, and $R_{1}$ and $R_{2}$ their respective radii. (b) Face-on orientation, showing the relevant angles, $\theta_{1}$ and $\theta_{2}$, used to compute the obscured area of the primary.

Fig. 9.- Constraints on the binary hypothesis. Predicted $\Delta \mathrm{J}_{e c l}$ during maximal eclipse is plotted versus period for $q<0.63 ; \mathrm{M}_{2}>5 \mathrm{M}_{\text {Jup }} ; \tau=0.5$ (solid line), 1.0 (dot-dashed line), and 5.0 (dashed line) Gyr; and (from top to bottom) $i=90^{\circ}, 80^{\circ}$, $70^{\circ}$, and $60^{\circ}$. The hatched area covers the parameter space probed by the J-band monitoring observations. 
TABLE 1

$\mathrm{H} \alpha$ Line Strengths.

\begin{tabular}{ccccc}
\hline \hline UT Date & Uncorrected \\
$(1)$ & $\begin{array}{c}\mathrm{t}_{\text {int }}{ }^{\mathrm{a}}(\mathrm{s}) \\
(2)\end{array}$ & $\begin{array}{c}\text { Corrected } \\
f_{H \alpha}{ }^{\mathrm{a}} \\
(4)\end{array}$ & $\begin{array}{c}\text { Corrected } \\
\log \left(\mathrm{L}_{H \alpha} / \mathrm{L}_{\text {bol }}\right) \\
(5)\end{array}$ \\
\hline 1999 July 16 & 4800 & $6.9 \pm 0.3$ & $6.9 \pm 0.3$ & -4.3 \\
1999 July 17 & 1800 & $1.6 \pm 0.5$ & $3.4 \pm 1.0$ & -4.6 \\
1999 July 18 & 1800 & $8.4 \pm 1.4$ & $6.8 \pm 1.1$ & -4.3 \\
2000 March 5 & 1800 & $2.9 \pm 0.3$ & $7.4 \pm 0.6$ & -4.3 \\
2001 February 20 & 2400 & $10.6 \pm 0.3$ & $7.9 \pm 0.2$ & -4.2 \\
\hline
\end{tabular}

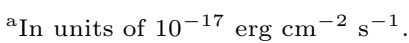

${ }^{\mathrm{b}}$ Data obtained in the $3800-8600 \AA$ regime.

TABLE 2

Log of IMAGing OBSERVATions.

\begin{tabular}{|c|c|c|c|c|}
\hline $\begin{array}{c}\text { UT Date } \\
\text { (1) }\end{array}$ & $\begin{array}{l}\text { UT Time } \\
(2)\end{array}$ & $\begin{array}{c}\text { Total t (hr) } \\
(3)\end{array}$ & $\begin{array}{l}\text { No. Images } \\
(4)\end{array}$ & $\begin{array}{c}\text { Conditions } \\
\text { (5) }\end{array}$ \\
\hline 19 May 2000 & $053126-080155$ & 2.51 & 155 & hazy and clear \\
\hline 20 May 2000 & $063628-091325$ & 2.62 & 165 & hazy with some scattered cirrus \\
\hline
\end{tabular}

TABLE 3

Parameters For the 2MASS 1237+6526 Binary Model.

\begin{tabular}{|c|c|c|c|c|c|}
\hline $\begin{array}{c}\tau(\mathrm{Gyr}) \\
(1)\end{array}$ & $\begin{array}{l}\alpha_{1}{ }^{\mathrm{a}} \\
(2)\end{array}$ & $\begin{array}{c}\mathrm{M}_{1}\left(\mathrm{M}_{J u p}\right) \\
(3)\end{array}$ & $\begin{array}{l}\alpha_{2}(\mathrm{mag})^{\mathrm{a}} \\
(4)\end{array}$ & $q_{\text {max }}^{\mathrm{b}}$ & $\begin{array}{c}M_{2, \max }\left(\mathrm{M}_{J u p}\right)^{\mathrm{b}} \\
(6)\end{array}$ \\
\hline 0.5 & 1.39 & 23 & 3.5 & $0.70^{\mathrm{c}}$ & 16 \\
\hline 1.0 & 1.36 & 30 & 3.3 & 0.53 & 16 \\
\hline 5.0 & 1.30 & 55 & 3.1 & 0.37 & 20 \\
\hline
\end{tabular}

${ }^{\text {a }}$ Scaling factor determined by comparison to the structure models of Burrows et al. (1997).

${ }^{\mathrm{b}}$ Maximum values permitted by non-detection of variability for $i>60^{\circ}$.

${ }^{\mathrm{c}}$ This value is greater than the maximum $q=0.63$ required to sustain mass loss. 


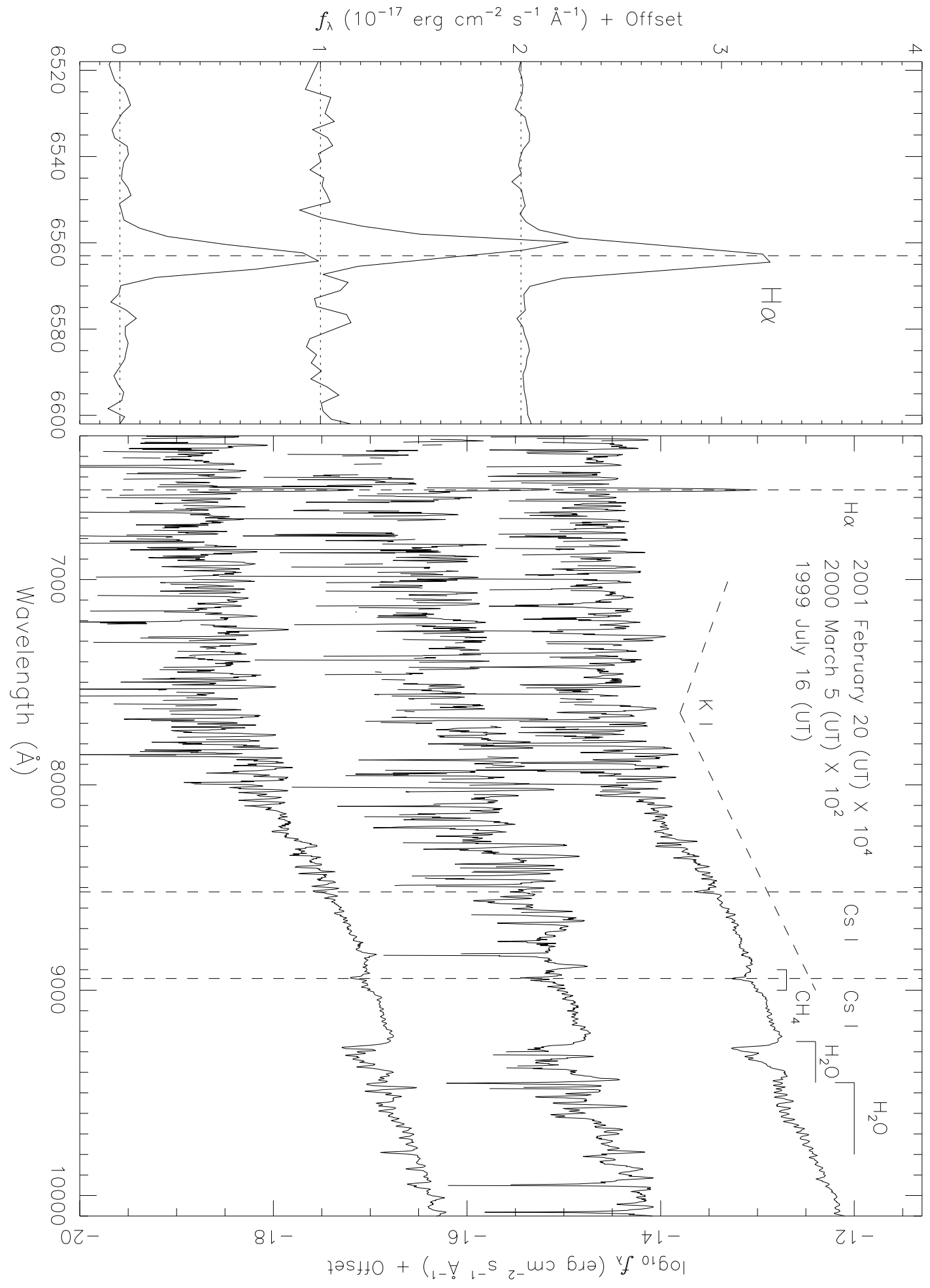

Fig. 1.- 


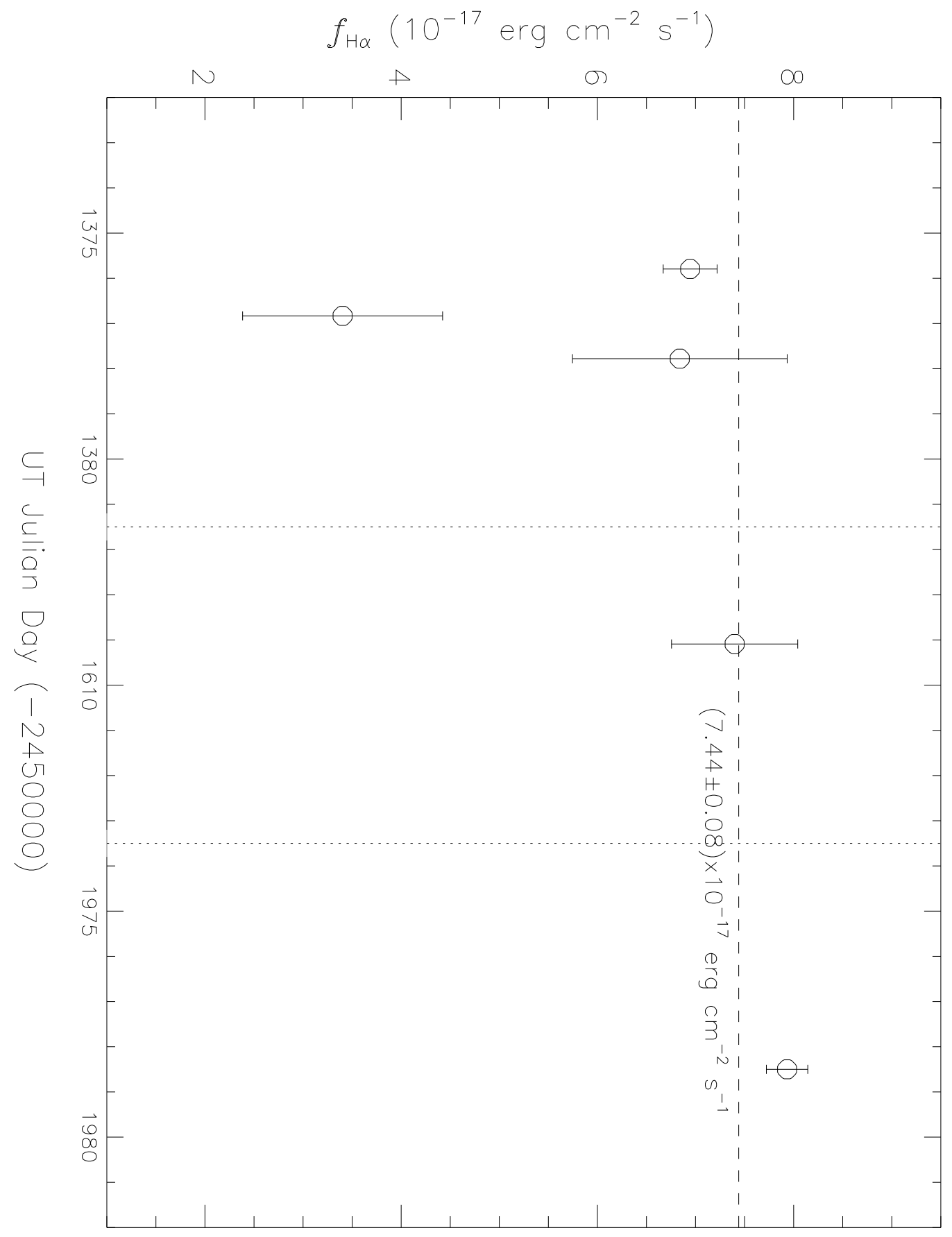

Fig. 2.- 


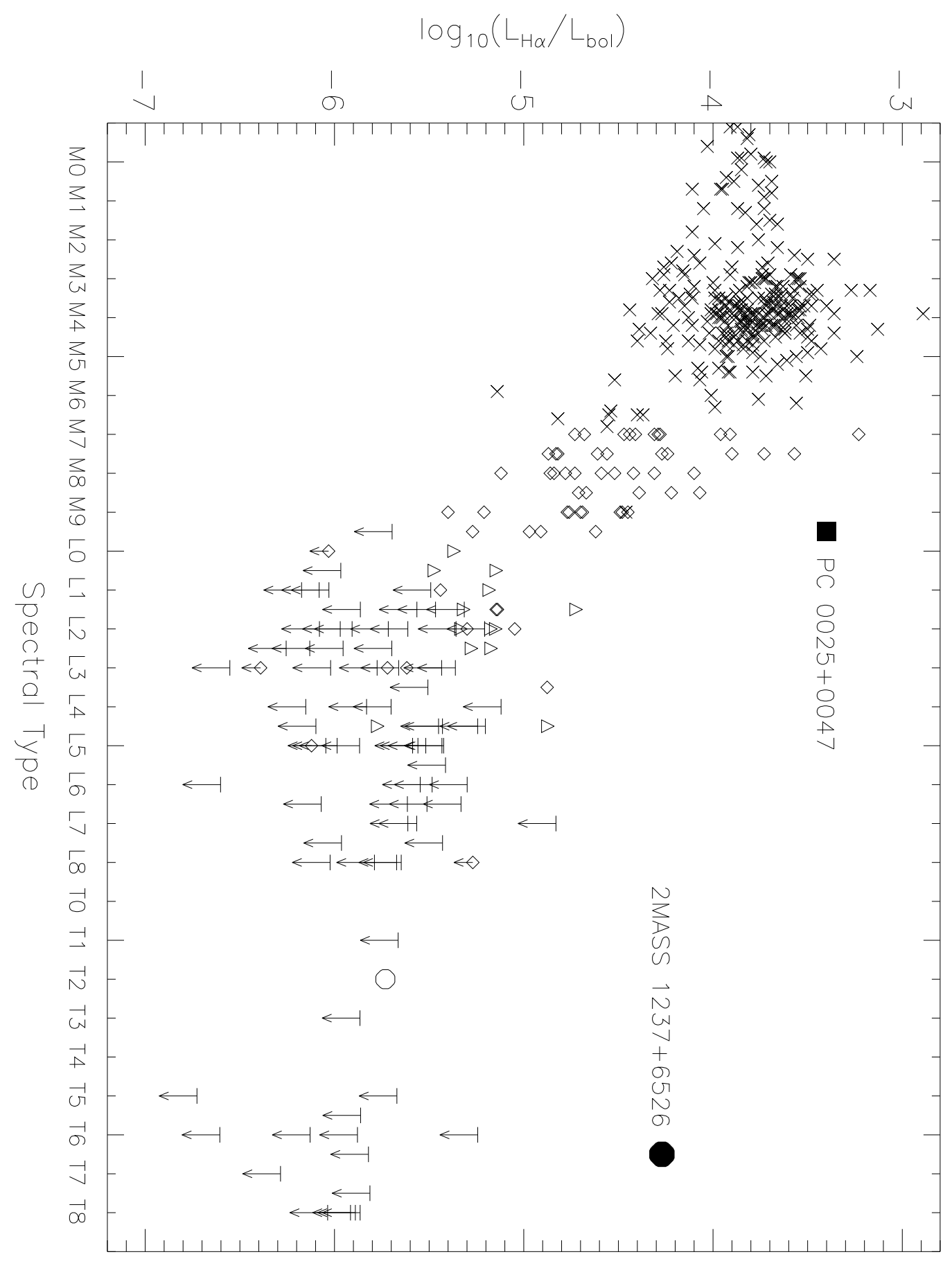

Fig. 3.- 

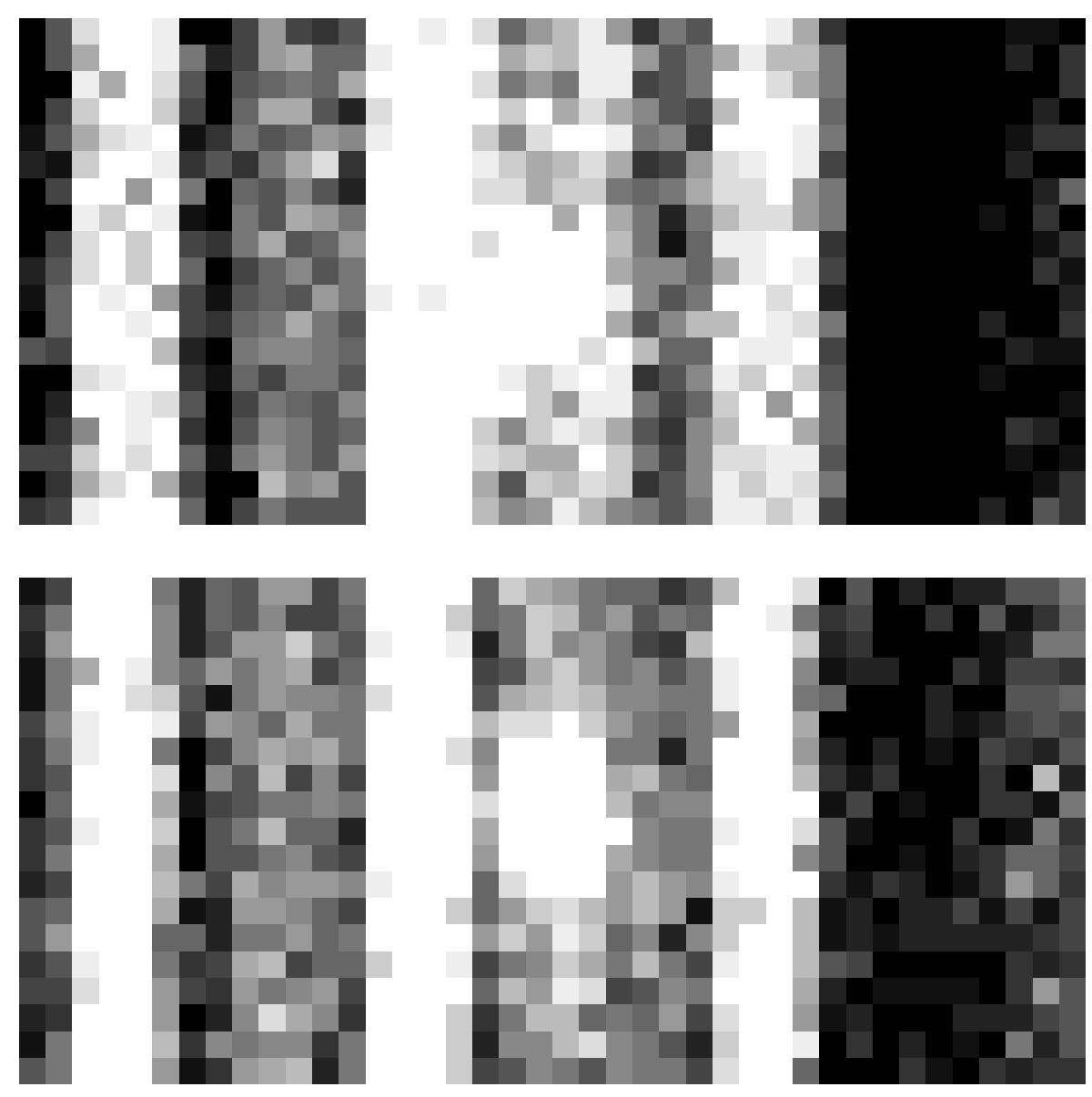

Fig. 4.- 


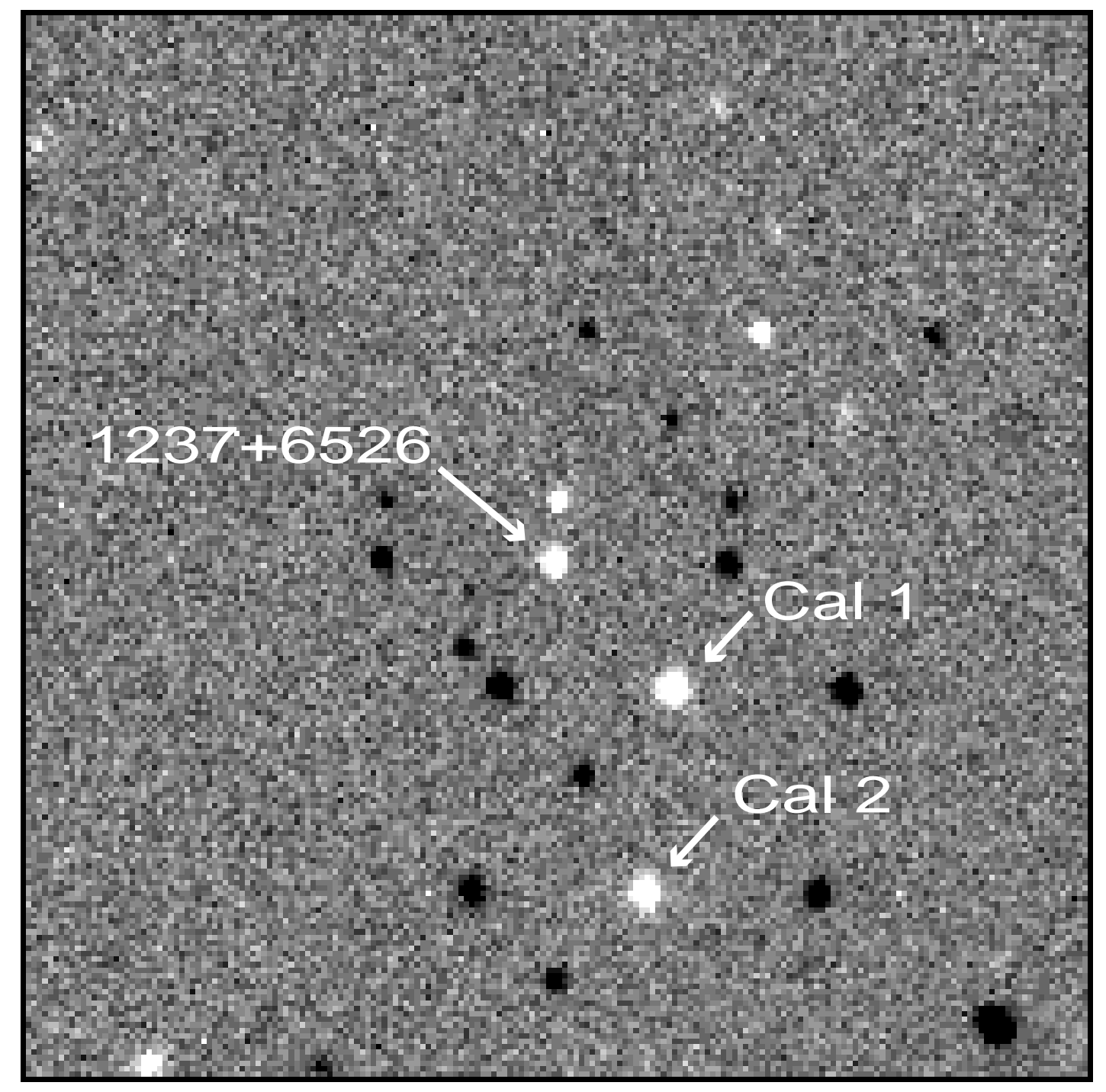

Fig. 5.- 


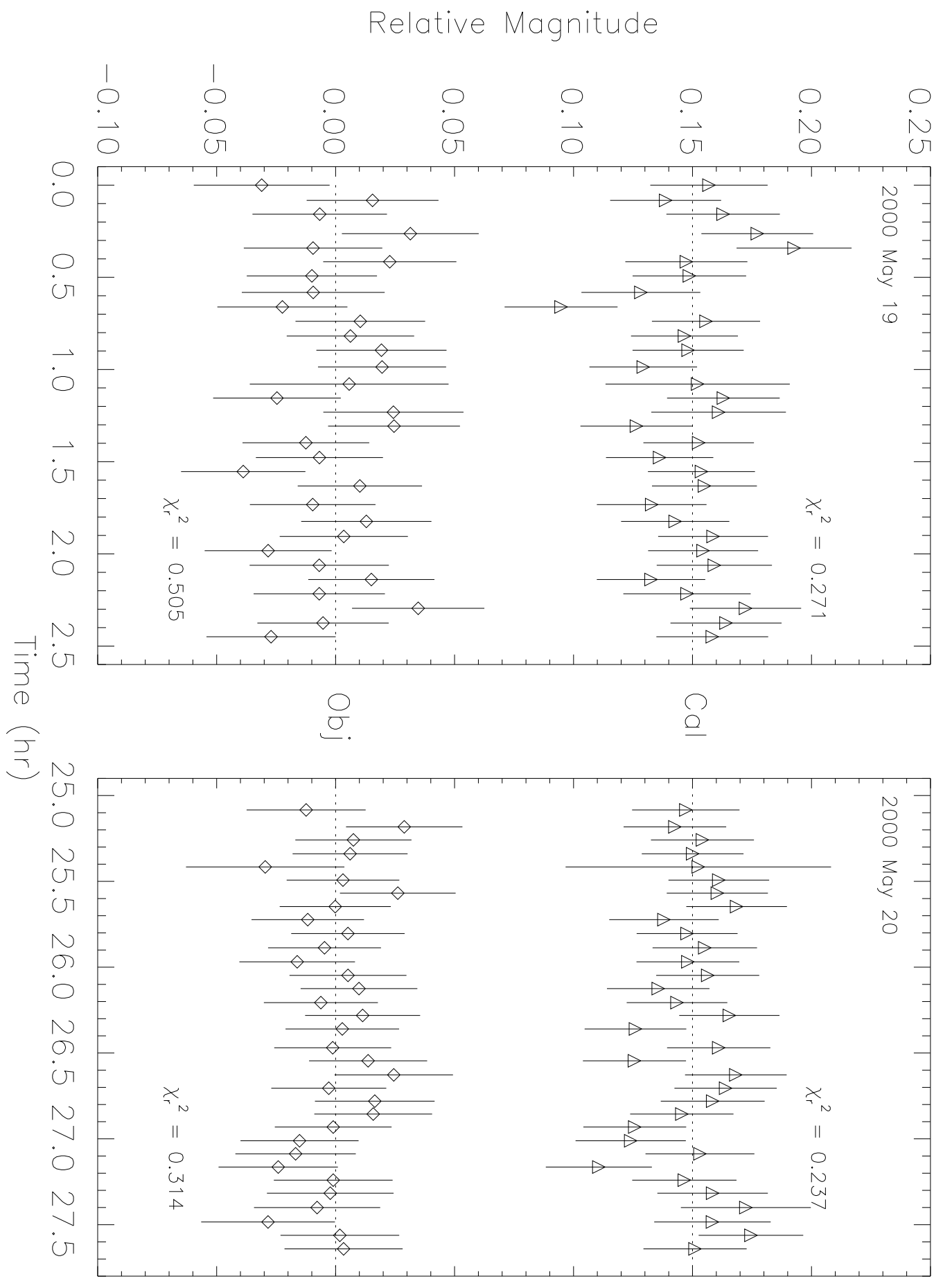

Fig. 6.- 


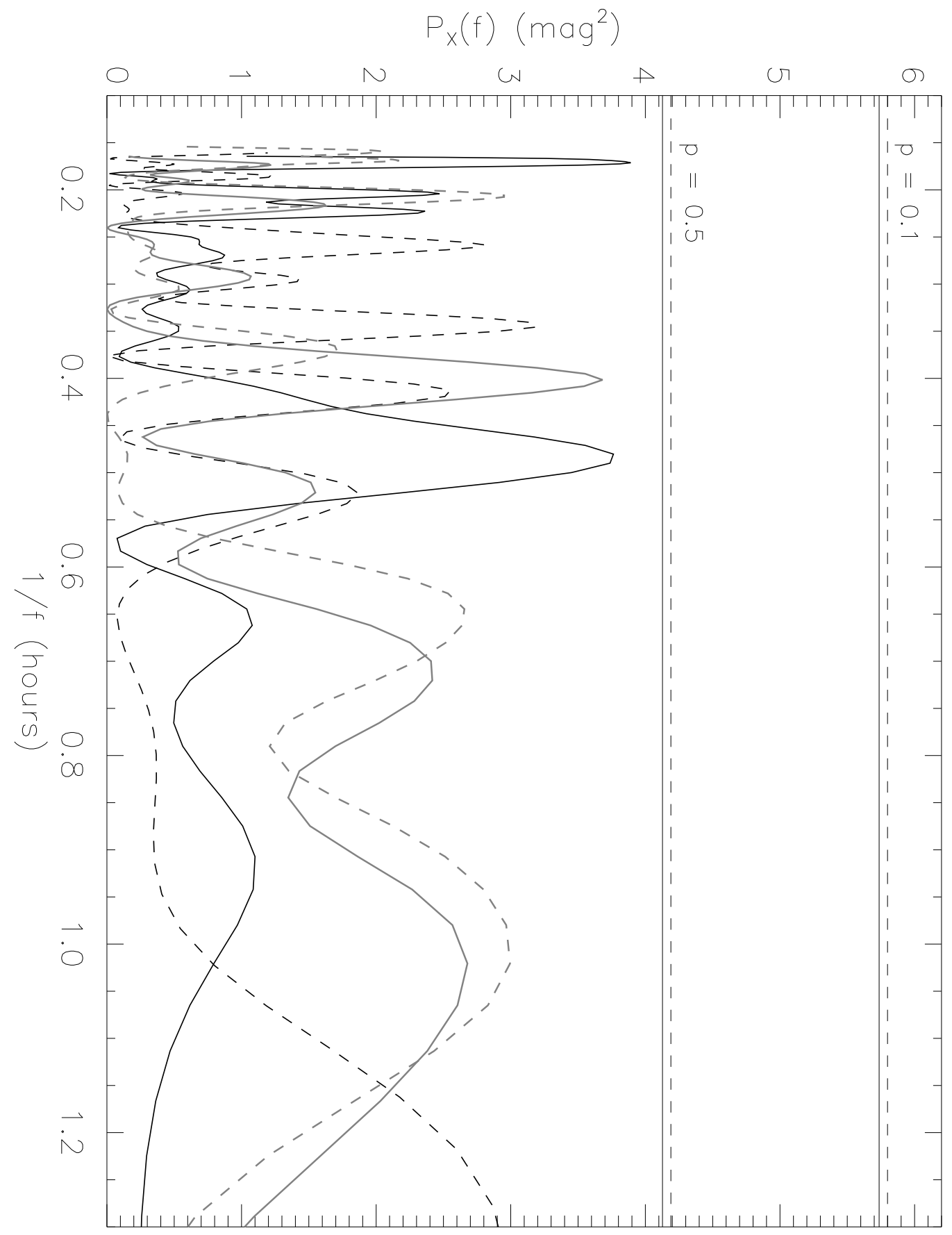

Fig. 7.- 

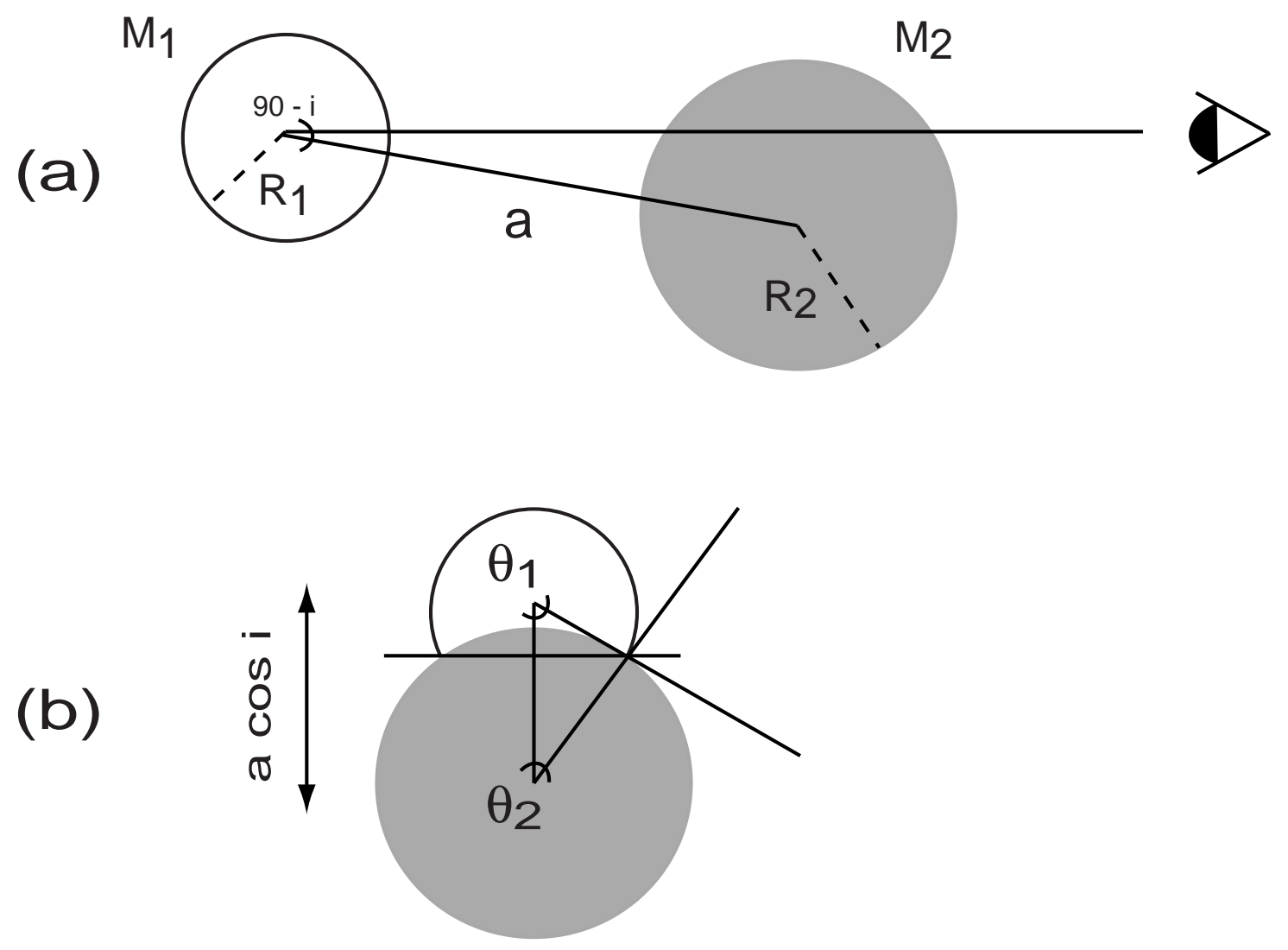

Fig. 8.- 


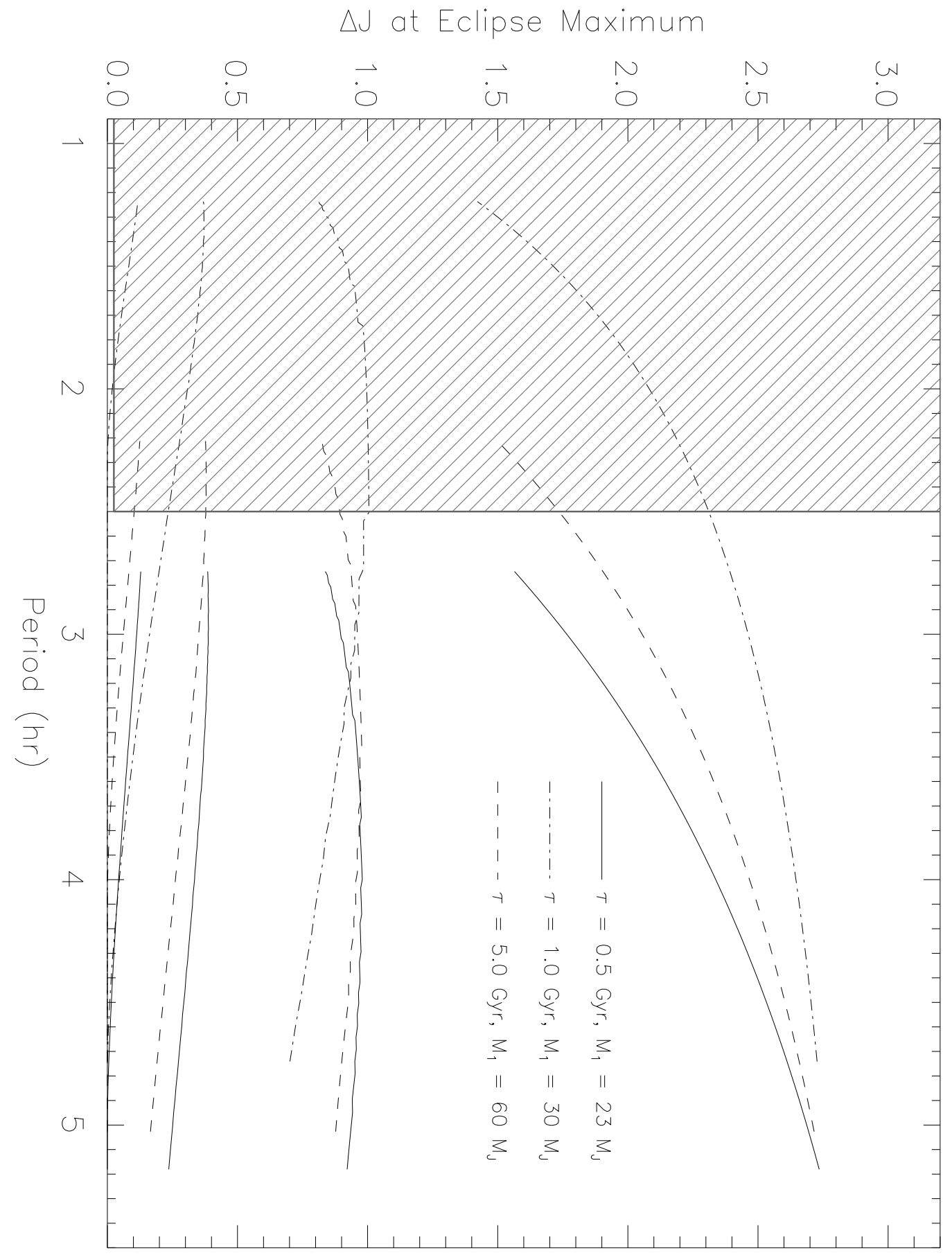

Fig. 9.- 